\title{
Synthesis and Photostability of Unimolecular Submersible Nanomachines: Toward Single-Molecule Tracking in Solution
}

Víctor García-López, ${ }^{\dagger}$ Jonathan Jeffet, ${ }^{\perp}$ Shunsuke Kuwahara, ${ }^{\dagger, \|}$ Angel A. Martí, ${ }^{\dagger}$ Yuval Ebenstein, ${ }^{*, \perp}$ and James M. Tour*, ${ }^{*},+, \S$

${ }^{\dagger}$ Department of Chemistry, ${ }^{\ddagger}$ NanoCarbon Center, ${ }^{\S}$ Department of Materials Science and NanoEngineering, Rice University, Houston, Texas 77005, United States

"Department of Chemistry, Faculty of Science, Toho University, 2-2-1 Miyama, Funabashi, Chiba 274-8510, Japan

${ }^{\perp}$ School of Chemistry, Raymond and Beverly Sackler Faculty of Exact Sciences, Tel Aviv University, Ramat Aviv 69978, Israel

\section{Supporting Information}

ABSTRACT: The synthesis and photophysical properties of a series of photostable unimolecular submersible nanomachines (USNs) are reported as a first step toward the analysis of their trajectories in solution. The USNs have a light-driven rotatory motor for propulsion in solution and photostable cy5-COT fluorophores for their tracking. These cy5-COT fluorophores are found to provide an almost 2-fold increase in photostability compared to the previous USN versions and do not affect the rotation of the motor.

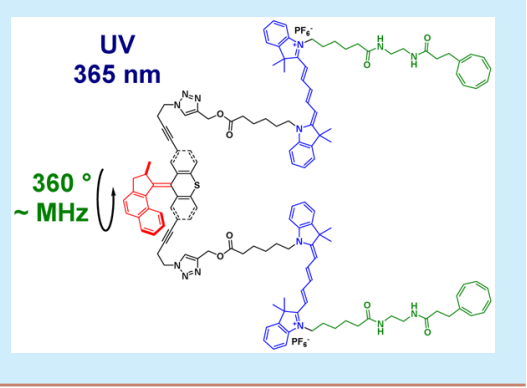

$\mathrm{D}$ uring the past three decades, synthetic chemists have been working on the development of artificial molecular machines capable of performing a variety of functions at the molecular level. ${ }^{1-3}$ Our group is particularly interested in developing single-molecule machines that convert external energy inputs into translational motion on surfaces (nanocars) ${ }^{4-6}$ or into enhanced diffusion in solution (submersible nanomachines $)^{7-9}$ with the ultimate goal of transporting cargoes from one place to another. This is similar to biological machines that transport important materials needed for different cellular functions. ${ }^{10}$

The main two hurdles in the area of submersible nanomachines are finding the appropriate propulsion mechanism that can overcome the Brownian motion and tracking the nanomachines. Recently, we demonstrated that unimolecular submersible nanomachines (USN-1, Figure 1) have their average diffusion in acetonitrile enhanced by $26 \%$ after light activation. ${ }^{7}$ The design included a light-driven motor that rotates unidirectionally at 2 to $3 \mathrm{MHz}$ at $25^{\circ} \mathrm{C}^{11}$ to propel the machines and the two cy5 fluorophores. This was measured in solution by fluorescence correlation spectroscopy (FCS). Cy5 was chosen because its absorption $(>630 \mathrm{~nm})$ is far from the motor absorption $(365 \mathrm{~nm})$, and therefore both can be excited at the same time without crosstalk. ${ }^{6,7}$

In contrast to FCS where approximately one molecule is excited at a time for short periods of time, analyzing the trajectories of the USN requires long excitation times of a high population of molecules. To allow discrimination of Brownian diffusion from propelled motion, it is estimated that at least 1.5 $\mathrm{s}$ of continuous single molecule detection and tracking is required (30 frames at $50 \mathrm{~ms}$ per frame). Under these conditions, photobleaching of the cy5 fluorophores severely impaired the tracking capability of the USN-1. This was presumably caused by photo-oxidation by singlet oxygen generated from reactions between molecular oxygen and the cy5 triplet excited state. ${ }^{12,13}$ The use of oxygen scavengers in order to enhance the photostability of the cy5 is not compatible with the organic medium used in this experiment. On the other hand, the addition of antibleaching additives ${ }^{14,15}$ to the medium resulted in aggregation of the USN-1 and had little effect on the photostability of the cy5 fluorophores.

Blanchard and co-workers reported that the covalent linkage of antibleaching additives to cy5 reduced photobleaching in both deoxygenated and oxygenated environments. ${ }^{16}$

The best results in oxygenated environments were obtained when cyclooctatetraene (COT, a triplet-state quencher) was covalently attached to cy5 fluorophores. In order to circumvent the photobleaching and aggregation problems of USN-1, the rotatory motor was adapted with modified cy5-COT fluorophores $^{16}$ (Figure 1).

The synthesis of the cy5-COT fluorophores began with an EDC coupling between COT-amine $4^{16}$ and acid $5^{17}$ to afford intermediate COT-amide 6 . The cy5 core was made in $29 \%$ yield over two steps from 4 by reacting COT-amide 6 with in situ prepared intermediate 7 in pyridine. Then, cy5-COT-acid 8 was reacted with propargyl alcohol to obtain cy5-COT-alkyne 9 (Scheme 1). The final step of the synthesis is a double azidealkyne Huisgen cycloaddition between cy5-COT-alkyne 9 and previously reported azide motor $10^{7}$ followed by ion exchange to afford photostable USN-2 (Scheme 2).

Received: March 15, 2016

Published: April 28, 2016 

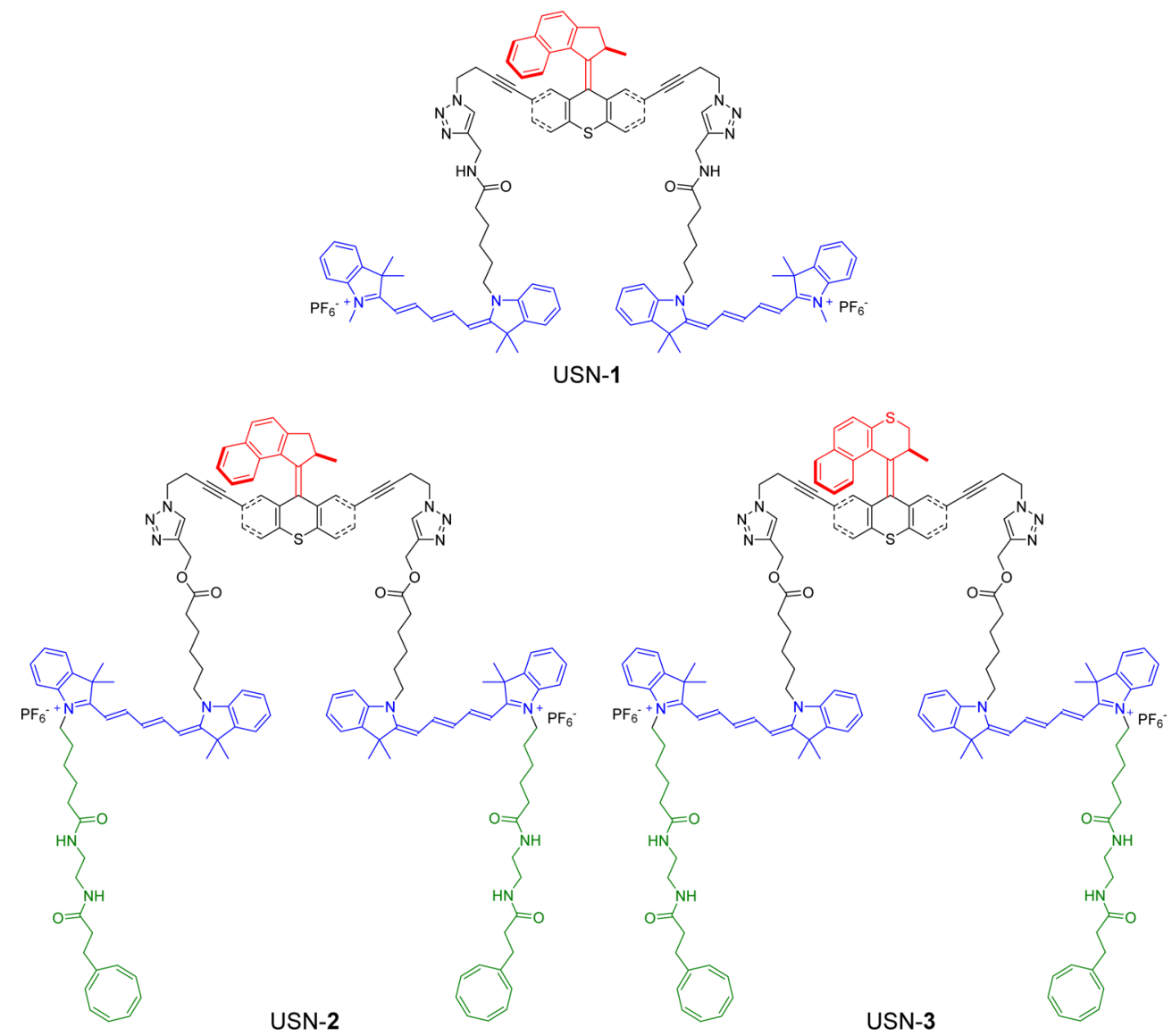

Figure 1. Previously reported USN-1 ${ }^{7}$ and new more photostable USN-2 and USN-3. The rotor portion is shown in red, the stator and spacers are in black, the fluorescent cyanine cores are in blue, and the COT-linkages are in green.

Scheme 1. Synthesis of cy5-COT-Alkyne
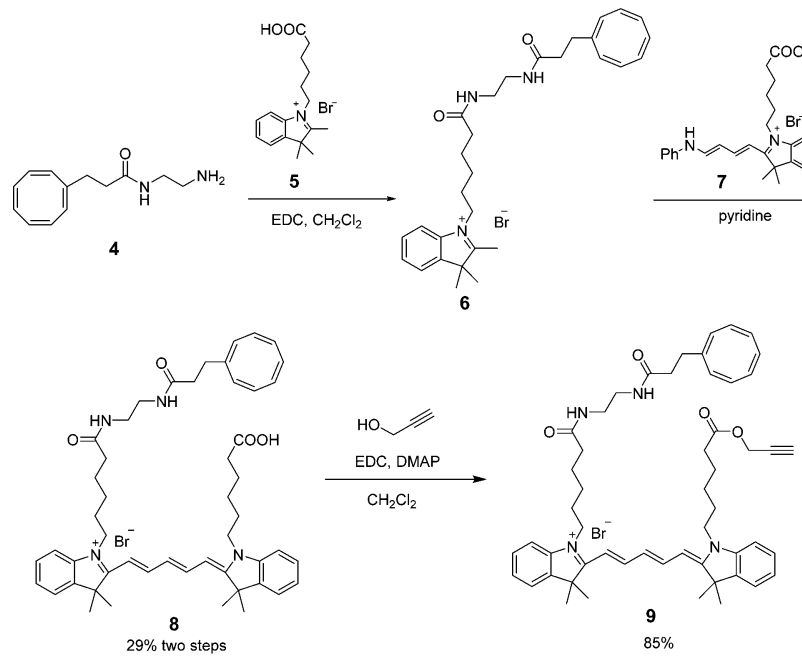

Scheme 2. Synthesis of Photostable USN-2

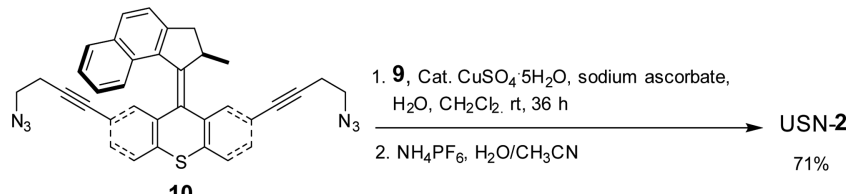

10

Due to the high frequency of USN-2, its rotation in the presence of the cy5-COT fluorophores cannot be monitored at room temperature by NMR. Therefore, the control molecule, USN-3, bearing a slow rotatory motor $\left(\sim 2 \mathrm{rph} \text { at } 60{ }^{\circ} \mathrm{C}\right)^{4}$ was analogously synthesized from 11 and 9. A $1 \mathrm{mM}$ sample of USN-3 in an NMR tube in $\mathrm{CD}_{3} \mathrm{CN}$ was irradiated at room temperature with $365 \mathrm{~nm}$ light for $1 \mathrm{~h}$. As shown by the ${ }^{1} \mathrm{H}$ NMR analysis, $88 \%$ of the stable isomer of USN-3 is converted to the unstable isomer (Figure 2). When the slow motor without fluorophores is excited at $365 \mathrm{~nm}$ under the same conditions, the unstable isomer is formed also in $88 \%$ yield, suggesting that the cy5-COT does not interfere with the photoisomerization of the motor just as the non-COTcontaining cy5 did not interfere. Then, unstable USN-3 was heated at $60{ }^{\circ} \mathrm{C}$ for $1 \mathrm{~h}$ to drive the thermal helix inversion and to obtain the stable isomer. The chemical shifts returned to the original values, completing a half-rotation of the motor without appreciable photodecomposition or thermal decomposition. After another photoisomerization and thermal helix inversion, the motor completes 1 cycle. This study indicates that the incorporation of the cy5-COT fluorophores does not affect the rotation of the motor and therefore the molecules can be propelled in solution in the same fashion as USN-1.

The optical properties of USN-1, USN-2, and USN-3 were investigated by $\mathrm{UV} /$ vis and fluorescent spectroscopy in $\mathrm{CHCl}_{3}$ solution (Table 1 and Figure S1). USN-1 has a maximum absorption at $656 \mathrm{~nm}$, corresponding to the cy5 moiety, while USN-2 and USN-3 exhibit a strong absorption at $660 \mathrm{~nm}$ (Figure S1). Unexpectedly, the quantum yields of USN-2 and USN-3 (0.38) are higher than the quantum yield of USN-1 


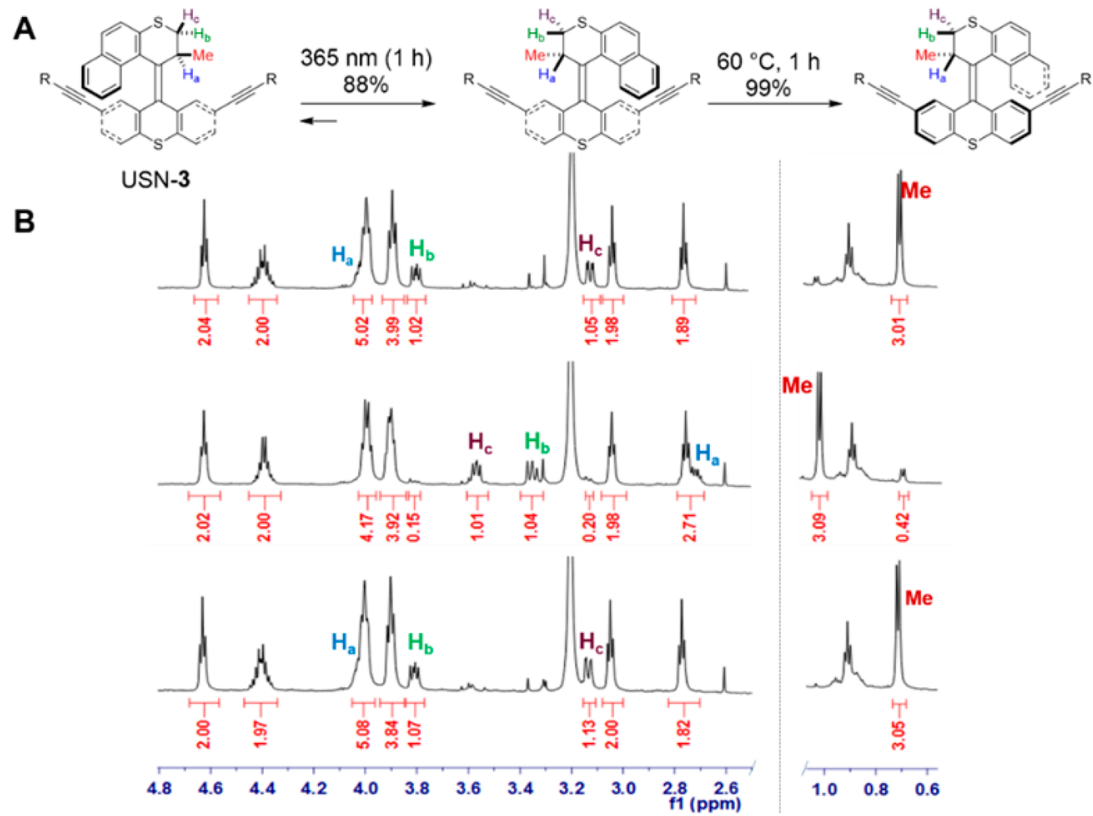

Figure 2. (A) Representation of the photoisomerization of the slow motor. (B) Partial ${ }^{1} \mathrm{H}$ NMR spectra of the photoisomerization study of USN-3 before (top) and after (middle) $1 \mathrm{~h}$ of UV irradiation at $365 \mathrm{~nm}$. After $1 \mathrm{~h}$ of heating at $60{ }^{\circ} \mathrm{C}$, the stable isomer (bottom) is formed, completing the half-rotation of the motor.

Table 1. Optical Properties ${ }^{a}$ of USN-1, USN-2, and USN-3

$\begin{array}{cccc}\text { compound } & \lambda_{\text {abs }}(\mathrm{nm}) & \lambda_{\text {em }}(\mathrm{nm}) & \Phi_{\mathrm{F}}{ }^{b} \\ \text { USN-1 } & 656 & 680 & 0.30 \\ \text { USN-2 } & 660 & 683 & 0.38 \\ \text { USN-3 } & 660 & 683 & 0.38\end{array}$

${ }^{a}$ Determined in $\mathrm{CHCl}_{3}$ solution. ${ }^{b}$ Calculated using Williams' method. ${ }^{18}$ Zinc phthalocyanine was used as a reference, $\Phi_{\mathrm{F}}=0.30^{19}$ in benzene, $\lambda_{\text {exc }}=630 \mathrm{~nm}$.

(0.30) even in solutions where $\mathrm{O}_{2}$ concentration was reduced by bubbling $\mathrm{N}_{2}$.

The improvement in quantum yield of UNS-2 is helpful because it has a higher photon count; the most critical property for single-molecule tracking is the photostability of the fluorophores. To study the photostability, USN-2 was dissolved in DMSO and deposited on clean coverslips for single-molecule imaging. Upon excitation at $637 \mathrm{~nm}$, surface-bound USN-2 was detected as fluorescent spots in the field of view $\left(80 \times 80 \mu \mathrm{m}^{2}\right)$. Time lapse movies were acquired for several hundreds of USN$2(N \approx 700)$ to assess their photostability (500 frames, $50 \mathrm{~ms} /$ frame, Figures S2 and S3). The time traces of the molecules exhibited mostly a single photobleaching step (molecules A, Figure 3) with occasional blinking events (molecule B, Figure 3 ), although in some cases two steps were evident (molecule $C$ in Figure 3) which corresponds to the photobleaching of the two cy5 fluorophores at different times.

The distribution of the fluorescence time before photobleaching between three time regimes is shown for USN-1 and USN-2 (Figures S4 and S5). The improved photostability of USN-2 gained by the incorporation of the cy5-COT is evident from the increased percentage of a long-lived fluorescence population. A total of 731 USN-1 molecules were analyzed, and only $23 \%$ have a total on-time $\left(\tau_{\text {on }}\right)$ higher than $1.5 \mathrm{~s}$. We found that this percentage is too low to obtain enough data for accurate discrimination of random Brownian diffusion from induced propelled motion. From 631 molecules analyzed of the

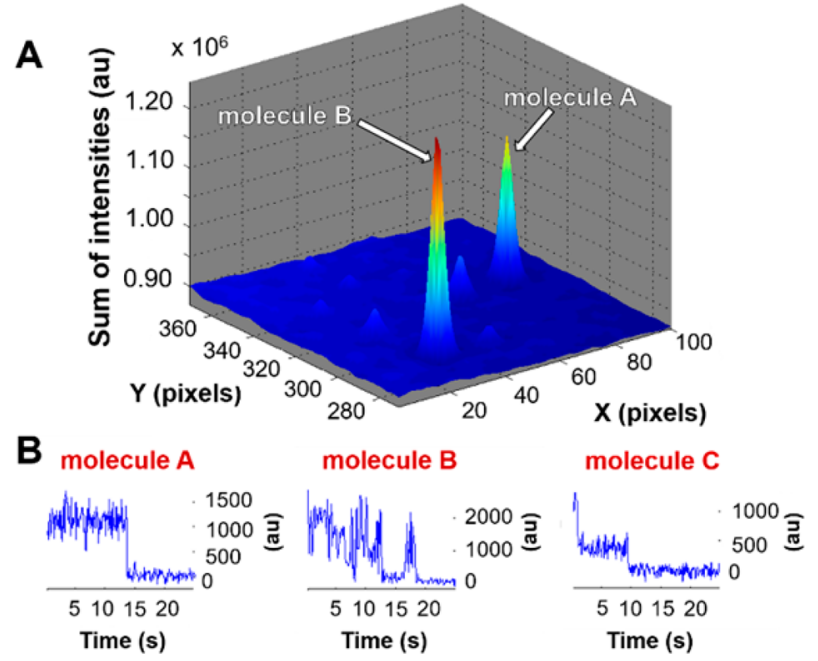

Figure 3. (A) 3D plot of fluorescence intensity across a section of the imaging surface depicting the fluorescence of two USN-2 molecules. (B) Time traces of three representative molecules showing the temporal behavior of fluorescence emission until photobleaching. Only molecules $\mathrm{A}$ and $\mathrm{B}$ are shown in the main $3 \mathrm{D}$ plot.

more photostable USN-2, 43\% had a total on-time $\left(\tau_{\text {on }}\right)$ higher than $1.5 \mathrm{~s}$. The population of molecules that have $\tau_{\text {on }}$ higher than $4 \mathrm{~s}$ is also higher for USN-2, 20\% versus $6 \%$ of the USN-1 population. This increased photostability is crucial for the success of future tracking experiments.

In order to numerically estimate the average detection time window for a single USN-2 molecule, the average overall fluorescent $\tau_{\text {on }}$ was calculated to give the mean total $\tau_{\text {on }}$. In addition, a histogram of the total $\tau_{\text {on }}$ was obtained and fitted to a standard exponential decay law. The reciprocal of the decay coefficient was taken as the mean decay time of the set of molecules (Figure S6). In both calculations, an almost 2-fold increase in $\tau_{\text {on }}$ was calculated in favor of USN-2, in agreement 
with previously published results for similar cy5-COT derivatives. $^{16}$

In conclusion, the synthesis of a new set of USNs bearing cy5-COT fluorophores and a 2 to $3 \mathrm{MHz}$ rotatory motor ${ }^{7,11}$ for propulsion in solution was completed. The incorporation of the photostable cy5-COT fluorophores resulted in a 2-fold enhancement in photostability, now making feasible the analysis of their trajectories by single-molecule tracking. By using ${ }^{1} \mathrm{H}$ NMR spectroscopy, it was demonstrated that the incorporation of the cy5-COT fluorophores does not quench the rotation of the motor, which has to keep its high rotatory action to propel the USN in solution. ${ }^{7}$ This new USN design will allow the investigation and understanding of the dynamic behavior of light-driven submersible nanomachines in solution through single-molecule tracking.

\section{ASSOCIATED CONTENT}

\section{S Supporting Information}

The Supporting Information is available free of charge on the ACS Publications website at DOI: 10.1021/acs.orglett.6b00506.

Detailed experimental procedures and spectroscopic data (PDF)

\section{AUTHOR INFORMATION}

\section{Corresponding Authors}

*E-mail: uv@post.tau.ac.il.

*E-mail: tour@rice.edu.

Notes

The authors declare no competing financial interest.

\section{ACKNOWLEDGMENTS}

J.M.T. and A.A.M. acknowledge support from the National Science Foundation (CHE-1007483). S.K. acknowledges support from Grant-in-Aid for Scientific Research (C) No. 25410100 and Supported Program for the Strategic Research Foundation at Private Universities (2012-2016) from MEXT of Japan, and Yamada Science Foundation. Y.E. acknowledges support from i-Core program of the Israel Science Foundation (Grant No. 1902/12) and the European Research Councils starter grant (Grant No. 337830).

\section{REFERENCES}

(1) Shirai, Y.; Morin, J.-F.; Sasaki, T.; Guerrero, J. M.; Tour, J. M. Chem. Soc. Rev. 2006, 35, 1043-1055.

(2) Credi, A.; Silvi, S.; Venturi, M. Molecular Machines and Motors. Recent Advances and Perspectives; Springer: 2014; pp 1-338.

(3) Erbas-Cakmak, S.; Leigh, D. A.; McTernan, C. T.; Nussbaumer, A. L. Chem. Rev. 2015, 115, 10081-10206.

(4) Morin, J.-F.; Shirai, Y.; Tour, J. M. Org. Lett. 2006, 8, 1713-1716.

(5) Chiang, P.-T.; Mielke, J.; Godoy, J.; Guerrero, J. M.; Alemany, L. B.; Villágomez, C. J.; Saywell, A.; Grill, L.; Tour, J. M. ACS Nano 2012, 6, 592-597.

(6) García-López, V.; Chu, P.-L.; Chiang, P.-T.; Sun, J.; Martí, A. A.; Tour, J. M. Asian J. Org. Chem. 2015, 4, 1308-1314.

(7) García-López, V.; Chiang, P.-T.; Chen, F.; Ruan, G.; Martí, A. A.j Kolomeisky, A. B.; Wang, G.; Tour, J. M. Nano Lett. 2015, 15, 82298239.

(8) Godoy, J.; García-López, V.; Wang, L.-Y.; Rondeau-Gagne, S.; Marti, A. A.; Link, S.; Tour, J. M. Tetrahedron 2015, 71, 5965-5972.

(9) Dey, K. K.; Pong, F. Y.; Breffke, J.; Pavlick, R.; Hatzakis, E.; Pacheco, C.; Sen, A. Angew. Chem., Int. Ed. 2016, 55, 1113-1117.
(10) Kolomeisky, A. B. Motor Proteins and Molecular Motors; CRC Press: 2015; pp 1-6.

(11) Klok, M.; Boyle, N.; Pryce, M. T.; Meetsma, A.; Browne, W. R.; Feringa, B. L. J. Am. Chem. Soc. 2008, 130, 10484-10485.

(12) Zheng, Q.; Jockusch, S.; Zhou, Z.; Blanchard, S. C. Photochem. Photobiol. 2014, 90, 448-454.

(13) Zheng, Q.; Jockusch, S.; Zhou, Z.; Altman, R. B.; Warren, J. D.; Turro, N. J.; Blanchard, S. C. J. Phys. Chem. Lett. 2012, 3, 2200-2203.

(14) Zheng, Q.; Juette, M. F.; Jockusch, S.; Wasserman, M. R.; Zhou, Z.; Altman, R. B.; Blanchard, S. C. Chem. Soc. Rev. 2014, 43, 10441056.

(15) Dave, R.; Terry, D. S.; Munro, J. B.; Blanchard, S. C. Biophys. J. 2009, 96, 2371-2381.

(16) Altman, R. B.; Terry, D. S.; Zhou, Z.; Zheng, Q.; Geggier, P.; Kolster, R. A.; Zhao, Y.; Javitch, J. A.; Warren, J. D.; Blanchard, S. C. Nat. Methods 2011, 9, 68-71.

(17) Kvach, V. M.; Ustinov, A. V.; Stepanova, I. A.; Malakhov, A. D.; Skorobogatyi, M. V.; Shmanai, V. V.; Korshun, V. A. Eur. J. Org. Chem. 2008, 2008, 2107-2117.

(18) Williams, A. T. R.; Winfield, S. A.; Miller, J. N. Analyst 1983, 108, 1067-1071.

(19) Brouwer, A. M. Pure Appl. Chem. 2011, 83, 2213-2228. 